\title{
Praxislabor und Patientensicherheit
}

\begin{abstract}
Das Praxislabor ist mit der Revision der Analysenliste durch das Bundesamt für Gesundheit (BAG) akut gefährdet und droht zu verschwinden. Mit der Revision will das BAG sparen - und zwar auf Kosten des Praxislabors und damit der Patientensicherheit. Gemäss dem BAG führt die derzeit in Diskussion stehende Revision zu Einbussen in der Höhe von 22 bis 25 Prozent. Unsere Berechnungen auf betriebswirtschaftlicher Basis ergeben weit höhere Einbussen, nämlich bis zu 36 Prozent. Das Praxislabor lässt sich somit nicht mehr kostendeckend betreiben. Es liegt jedem Arzt mit Praxislabor anheim, ob er sein Praxislabor mit erheblichen Mitteln durch die übrige Praxis querfinanzieren oder ob er die Analysen in ein Auftragslabor auslagern will.
\end{abstract}

\section{Der Verlust des Praxislabors gefährdet die Patienten- sicherheit, generiert eine Qualitätseinbusse und ist ein Verlust an Komfort für die Patienten}

Welches sind die Auswirkungen, wenn das Praxislabor vor allem im Grundversorgungsbereich verschwindet? Die Hausärztin und der Hausarzt benötigen das Praxislabor für die tägliche Arbeit. Es ist für den Praxisarzt ein Werkzeug, das ihm hilft, Sachverhalte zu objektivieren, und das zur Entscheidungsfindung für Diagnose und Therapie wesentlich beiträgt. Die Vergabe der Analysen an auswärtige Labors hat einschneidende Konsequenzen. Der Verlust dieses Praxislabors gefährdet die Patientensicherheit, generiert eine Qualitätseinbusse und ist ein Verlust an Komfort für die Patienten. Lange Wartezeiten auf die Diagnosen - die Resultate liegen ja erst am nächsten Tag vor -, verzögerte Therapieentscheide, unnötige Behandlungen, unnötige Hospitalisationen und zusätzliche Arztbesuche für die Patienten sind die Folge. Die Notfallversorgung in entlegenen Gebieten ist ohne Praxislabor nicht mehr gewährleistet. Die isolierte Betrachtung des Praxislabors durch das BAG führt zum Sparen am falschen Ort, zur Verlagerung von Kosten und insgesamt zu einer Verteuerung. Das gut funktionierende System der Hausarztpraxis wird zerstört und dem Grundversorger wird ein wichtiges Instrument seiner Tätigkeit weggenommen, was die Attraktivität des Berufes mindert.

Die FMH hat schon wiederholt darauf hingewiesen, dass die Präsenztaxe, mit der das BAG die Nachteile des Praxislabors gegenüber den Auftragslabors kompensieren will, ein absoluter Unsinn ist. Sie wird als Pauschale der grossen Varianz der verschiedenen medizinischen Spezialitäten - Pädiater, Grundversorger, Spezialisten verschiedener Herkunft - mit sehr unterschiedlichem Analysenspektrum und unterschiedlichen Analysenzahlen überhaupt nicht gerecht.
Die FMH hat in enger Zusammenarbeit mit Anton Prantl (Ärztekasse) und Roman Fried (Verein für medizinische Qualitätskontrolle Zürich) auf der Basis der Präsenzdiagnostik ein eigenes Modell (Point-of-Care-Diagnostik) entwickelt, das auf der Basis der Work-Flow-Analyse sauber und betriebswirtschaftlich gerechnet die Aufwendungen des Praxislabors (Löhne, Gerätekosten, Reagenzienkosten usw.) abbildet. Leitidee des Modells ist es, die Laboranalysen, die in Anwesenheit des Patienten gemacht werden, als separate Analyse im Tarif abzubilden. Das heisst, dass es z. B. eine Position «CRP» und eine Position «CRP poc» gibt. Wir haben das Modell «Point-of-Care-Diagnostik» und Probleme mit der Präsenztaxe dem BAG am 30. Oktober 2008 vorgestellt. Der definitive Entscheid des BAG steht noch aus. Aus unseren Berechnungen resultiert im Vergleich zur heutigen Situation ein Nullsummenspiel. Das heisst, für das BAG resultiert kein Einsparpotential für das Praxislabor. Es entsteht aber auch kein Kostenschub.

Wir appellieren an das BAG, sich seiner Verantwortung bewusst zu werden. Das Praxislabor wird zusammenbrechen, das Know-how verfallen, die Patientensicherheit ist gefährdet und neue Arztgehilfinnen werden in der Labortätigkeit gar nicht mehr ausgebildet

Wie weiter? Das BAG hat die Einführung gemäss Medienmitteilung auf unbestimmte Zeit verschoben! In Unkenntnis des Entscheides des BAG sowie aufgrund der

\section{Die isolierte Betrachtung des Praxislabors durch das BAG führt zum Sparen am falschen Ort}

Annahme, dass die Präsenztaxe beibehalten wird und das BAG auf dem Sparpotential beharren wird, hat die Delegiertenversammlung der FMH am 19. November 2008 einstimmig beschlossen, einen Massnahmenkatalog zu öffentlichen Manifestationen unter der Führung der FMH umzusetzen. Der Massnahmenkatalog wurde breit abgestützt entwickelt (AG Labor, Fachgesellschaften, kantonale Ärztegesellschaften) und beinhaltet verschiedene Ebenen. Es sind Massnahmen auf technischer, politischer und medialer Ebene sowie Massnahmen mit der Basis geplant. Ziel dieser Massnahmen ist es, eine Neuaufgleisung der Revision Analysenliste zu erreichen. Diese Massnahmen werden nach einem vorgegebenen Zeitplan und aufgrund von Priorisierungen mit der gesamten Ärzteschaft umgesetzt werden. «Gemeinsam sind wir stark.»

Dr. med. Ernst Gähler, Vizepräsident der FMH, Leiter Ressort Tarife und Verträge 